\section{Utilização da insulina glargina em crianças menores de oito anos de idade}

\author{
Use of insulin glargine in type 1 diabetes children \\ with less than eight years old
}

Guilherme Rollin', Márcia Punales', César Geremia', Gislaine Vissoky $\mathrm{Ce}^{1}$, Balduino Tschiedel ${ }^{1}$

\begin{abstract}
RESUMO
Objetivos: Avaliar prospectivamente a eficácia e a segurança da insulina glargina no controle metabólico de crianças com diabetes melito tipo 1 (DMT1) com menos de oito anos de idade. Métodos: Foram avaliados 19 meninos e 11 meninas. Antes de iniciar a insulina glargina, todas as crianças foram colocadas em tratamento intensivo com insulina NPH e insulina asparte durante três meses. Posteriormente, os pacientes foram acompanhados por 12 meses para o tratamento com glargina. Todos os pacientes realizavam medidas da glicemia capilar 3-7 vezes ao dia. Desfechos principais: controle metabólico por meio da hemoglobina glicada (A1c); ocorrência de hipoglicemia leve (glicemia capilar $<60 \mathrm{mg} / \mathrm{dL}$ ) e ocorrência de hipoglicemia grave (perda ou alteração na consciência, convulsão ou necessidade de intervenção médica). Resultados: A1c média no início do estudo foi 8,68\%, semelhante ao valor obtido ao final dos 12 meses de tratamento com glargina $(8,64 \% ; p=0,82)$. A frequência de hipoglicemia leve às 3 horas da madrugada foi $1,43 / 3$ meses por paciente com insulina NPH e de $0,28 / 3$ meses por paciente com insulina glargina $(p<0,007)$. Em relação à hipoglicemia severa, houve uma diferença favorável à glargina: 0,008 versus 0,56 eventos $/ 3$ meses por paciente $(p<0,002)$. Conclusões: 0 uso da insulina glargina no tratamento de crianças com DMT1 foi considerado tão eficaz quanto o uso da NPH, apresentando, no entanto, melhor perfil de segurança caracterizado pelo menor risco de hipoglicemia noturna e severa. Arq Bras Endocrinol Metab. 2009;53(6):721-5
\end{abstract}

Descritores

Diabetes melito tipo 1; insulina glargina; criança; hipoglicemia

\begin{abstract}
Objectives: To evaluate prospectively the efficacy and safety of insulin glargine use for the metabolic control of type 1 diabetes mellitus (T1DM) children younger than eight years old. Methods: Nineteen boys and 11 girls with T1DM were included. Before initiating insulin glargine, all children received intensive NPH and aspart insulins for three months. Afterwards, they were assisted for 12 more months for glargine treatment. All patients performed self blood glucose monitoring before and two hours after meals and in early morning (3:00 AM). Primary endpoints: metabolic control using A1C levels; frequency of mild hypoglycemia (capillary glyce$\mathrm{mia}<60 \mathrm{mg} / \mathrm{dL}$ ); and frequency of severe hypoglycemia (loss or alteration of consciousness, seizures or need for medical intervention). Results: Mean A1C at the study entry was $8.68 \%$ and after 12 months of glargine, was $8.64 \%$ ( $p=0.82$ ). Frequency of mild hypoglycemia at 3.00 AM was $1.43 / 3$ months during the NPH period and $0.28 / 3$ months during the glargine period ( $p<0.007$ ). Frequency of severe hypoglycemia was $0.56 / 3$ months during the NPH period and $0.008 / 3$ months during the glargine period $(p<0.002)$. Conclusions: The treatment of T1DM children with insulin glargine was considered as efficacious as with NPH. However, a better safety profile, disclosed by the lower incidence of nocturnal and severe hypoglycemia episodes, was observed for insulin glargine. Arq Bras Endocrinol Metab. 2009;53(6):721-5
\end{abstract}

Keywords

Type 1 diabetes mellitus; insulin glargine; child; hypoglycemia
1 Instituto da Criança com Diabetes (ICD), Grupo Hospitalar Conceição, Porto Alegre, RS, Brasil

Correspondência para:

Balduino Tschiede

Instituto da Criança com Diabetes Rua Álvares Cabral, 529 - Cristo Redentor

91350-250 - Porto Alegre, RS, Brasil tbalduino@ghc.com.br

Recebido em 22/Jan/2009 Aceito em 29/Mar/2009 


\section{INTRODUÇÃO}

A insulina glargina é um análogo da insulina que permite um melhor perfil de insulina basal, sem picos e, consequentemente, com menor risco de hipoglicemia. A insulina glargina é capaz de proporcionar uma concentração basal de insulina constante e confiável com duração de 24 horas (1-5). Essa insulina é sintetizada a partir de modificações na cadeia de aminoácidos da insulina humana, efetuando-se uma substituição da asparagina por glicina na posição A2l e adição de duas argininas na posição B30. Essas modificações resultam em um padrão único de liberação a partir do local de injeção. Ou seja, esse análogo precipita-se no tecido subcutâneo, permitindo uma absorção gradual para a corrente sanguínea. Essas características explicam as diferenças farmacocinéticas da insulina glargina em comparação à NPH e ultralenta (3). Assim, a glargina permite uma ação aproximada de 24 horas, sem picos e com variação interindividual semelhante à observada no tratamento com bomba de infusão contínua de insulina subcutânea e significativamente menor do que a da NPH e ultralenta $(1-3,5)$.

Vários estudos demonstraram os benefícios do uso da insulina glargina quando comparada à $\mathrm{NPH}$, tanto na melhora do controle glicêmico como na redução dos episódios de hipoglicemia, principalmente os episódios noturnos (6-14), que são bastante frequentes em crianças com menos de oito anos de idade. Adicionalmente, esse perfil mais estável da glargina permite uma administração mais flexível em relação aos locais e horários. $\mathrm{E}$, ainda, por ser uma solução, não precisa ser homogeneizada como a NPH, o que facilita ainda mais o seu manuseio pelo paciente. Os efeitos do tratamento com a insulina glargina no controle glicêmico e na ocorrência de hipoglicemia já estão bem documentados tanto para o diabetes tipo 2 quanto para o tipo 1. Porém, seu uso em pacientes pediátricos, especialmente crianças com idade inferior a seis ou oito anos, ainda não está bem estabelecido, sendo escassos os estudos nessa população. Recentemente, Dixon e cols. (15) publicaram um estudo retrospectivo que demonstrou uma redução significativa nas hipoglicemias severas noturnas em crianças com menos de seis anos de idade que utilizavam a insulina glargina. Entretanto, informações a partir de estudos prospectivos ainda não estão disponíveis na literatura.

Neste estudo prospectivo, avaliaram-se a eficácia e a segurança da insulina glargina no controle metabólico de crianças menores de oito anos de idade com diabetes melito tipo l (DMTl). Nesse contexto, comparou-se a insulina glargina à NPH, especialmente em relação à ocorrência de hipoglicemias.

\section{PACIENTES E MÉTODOS}

Foram alocadas para o estudo crianças portadoras de DMTl com menos de oito anos de idade atendidas no Instituto da Criança com Diabetes (ICD). Essas crianças apresentavam diagnóstico de DMTl há pelo menos seis meses e mediam glicemia capilar de três a sete vezes ao dia.

Antes de iniciar o uso da insulina glargina, a criança permaneceu por três meses em acompanhamento intensivo para se ajustarem as doses de NPH e insulina ultrarrápida da melhor maneira possível. Foi oferecido tratamento por um ano com insulina glargina a todas as crianças que preencheram os critérios de inclusão em substituição à insulina NPH. O esquema de troca de doses de NPH para glargina foi o seguinte: mesma dose para casos de NPH administrada uma vez ao dia; $80 \%$ da dose da NPH para casos de administração de duas a três vezes ao dia. A insulina glargina era administrada sempre pela manhã, antes do café da manhã.

$\mathrm{O}$ ajuste da dose da insulina glargina seguiu o seguinte protocolo: aumento ou redução da dose de acordo com glicemia de jejum, objetivando-se uma glicemia entre 80 e $150 \mathrm{mg} / \mathrm{dL}$. Para crianças que necessitaram utilizar insulina rápida/ultrarrápida, foi fornecida a insulina asparte $\left(\right.$ Novorapid $\left.^{\circledR}\right)$, sendo as doses ajustadas conforme a glicemia capilar. $\mathrm{O}$ ajuste da dose da insulina asparte foi estabelecido conforme glicemias pré-prandiais, objetivando-se valores entre 100 e $200 \mathrm{mg} / \mathrm{dL}$ após as refeições, com esquema individualizado para cada caso.

A monitorização da glicemia capilar foi realizada por meio de medidas de glicemias capilares diariamente antes do café da manhã, almoço e jantar. Uma vez por semana, os pacientes e familiares foram orientados a realizar medidas adicionais da glicemia capilar às 3 horas da madrugada e pós-prandiais (duas horas após o café da manhã, almoço e jantar).

As consultas com endocrinologista foram mensais nos primeiros seis meses do estudo (três meses com NPH e primeiros três meses com glargina) e trimestrais a partir do sexto mês. Consultas com nutricionistas e demais membros da equipe multidisciplinar ocorreram de acordo com a rotina. Os pacientes foram orientados a entrar em contato por telefone caso a glicemia capilar permanecesse fora do alvo. 
Os desfechos avaliados foram: 1) controle metabólico avaliado pela hemoglobina glicada (AlC) a cada três meses; 2) hipoglicemia leve (glicemia capilar $<60 \mathrm{mg} / \mathrm{dL}$ ) na madrugada e antes do café da manhã; 3 ) hipoglicemia grave (perda ou alteração de consciência, crise convulsiva, necessidade de intervenção médica).

Este trabalho foi aprovado pela Comissão de Ética em Pesquisa do Grupo Hospitalar Conceição, com termo de consentimento livre e esclarecido assinado pelos pais das crianças que participaram do estudo.

\section{Análise estatística}

Os dados foram avaliados por meio da análise de variância (ANOVA) por medidas repetidas (comparações dos momentos da hemoglobina glicada) e do teste $t$ pareado para comparação da hemoglobina glicada e do número de hipoglicemias durante os dois tratamentos. Considerou-se significativo $\mathrm{p}<0,05$.

\section{RESULTADOS}

Foram avaliadas 30 crianças com DMTl, sendo 19 meninos e 11 meninas. A média de idade dos pacientes foi de 5,35 anos, variando de 3 a 7,5 anos. O tempo médio de diagnóstico do diabetes foi de dois anos e um mês, variando de oito meses a três anos. A média da Alc no início do estudo foi de 8,68\% (tratamento com NPH), valor semelhante ao encontrado no final do estudo: $8,64 \%$ (tratamento com glargina; $\mathrm{p}=0,82$ ) (Figura 1 ). A comparação múltipla pela análise de variância entre as médias da Alc (Alc durante o tratamento com NPH, sendo considerada a média entre tempo - 3 e 0 meses, e Alc durante tratamento com glargina avaliada nos meses 3, 6, 9 e 12) não evidenciou diferença estatisticamente significativa $(\mathrm{p}=0,18)$ (Tabela 1$)$.

A incidência de eventos hipoglicêmicos leves antes do café da manhã foi semelhante nos dois grupos: 7,77 eventos por paciente por três meses no período de tratamento com insulina NPH e 7,79 eventos por paciente durante os três meses de tratamento com glargina $(\mathrm{p}=0,99)$. Por outro lado, a incidência de hipoglicemias leves na madrugada e hipoglicemias graves foi muito maior durante o uso da insulina NPH ( $\mathrm{p}=0,007$ e $\mathrm{p}=0,002$, respectivamente). Foram registradas 1,43 hipoglicemia leve na madrugada e 0,56 hipoglicemia grave por paciente por três meses no período de tratamento com NPH e apenas 0,28 e 0,008 , respectivamente, no período com glargina (Tabela 2 ).

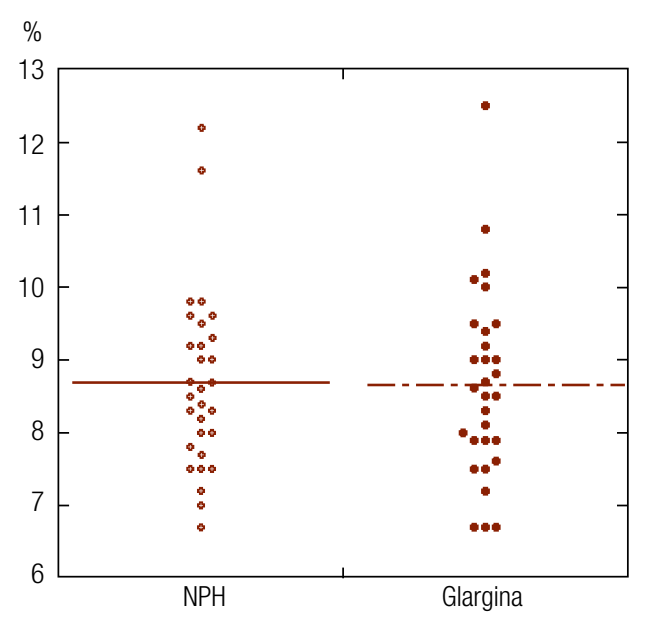

Figura 1. Valores da hemoglobina glicada (A1c) na primeira (-3 meses/NPH) e na última avaliação (12 meses/glargina): A1c NPH 8,68\% versus glargina 8,64\%; $p=0,82$.

\begin{tabular}{cccccc}
\hline $\begin{array}{l}\text { Tabela 1. Evolução da hemoglobina glicada ao longo do estudo (média } \pm \\
\text { desvio-padrão) }\end{array}$ \\
\begin{tabular}{cccccc} 
A1c (\%) NPH \\
\hline $\begin{array}{c}\mathbf{- 3} \text { e 0 } \\
\text { meses }^{\star}\end{array}$ & $\begin{array}{c}\mathbf{3} \\
\text { meses }\end{array}$ & $\begin{array}{c}\mathbf{6} \\
\text { meses }\end{array}$ & $\begin{array}{c}\mathbf{9} \\
\text { meses }\end{array}$ & $\begin{array}{c}\mathbf{1 2} \\
\text { meses }\end{array}$ & p $^{\star \star}$ \\
\hline 8,44 & 8,94 & 9,19 & 8,91 & 8,64 & 0,18 \\
$( \pm 1,12)$ & $( \pm 1,31)$ & $( \pm 1,32)$ & $( \pm 1,3)$ & $( \pm 1,29)$ & \\
\hline
\end{tabular}
\end{tabular}

* Média A1c tempos -3 e 0 meses.

${ }^{* *}$ ANOVA.

Tabela 2. Episódios de hipoglicemia nos períodos de tratamento com insulinas NPH e glargina (média por paciente em três meses)

\begin{tabular}{lccc}
\hline & NPH & Glargina & p \\
\hline Hipoglicemia grave & 0,56 & 0,08 & 0,002 \\
Hipoglicemia leve (3h) & 1,43 & 0,28 & 0,007 \\
Hipoglicemia leve (AC) & 7,77 & 7,79 & 0,99 \\
\hline
\end{tabular}

3h: às 3 horas da madrugada.

AC: antes do café da manhã.

\section{DISCUSSÃO}

$\mathrm{O}$ tratamento com insulina glargina permite um bom controle metabólico com menor incidência de hipoglicemia nos pacientes com DMTl, especialmente hipoglicemias noturnas $(6,10,14)$. Esses benefícios estão bem documentados em crianças maiores e em adolescentes $(6,10-12,14)$, porém estudos em menores de seis e oito anos de idade são escassos. Nossos resultados confirmam o benefício do tratamento com a insulina glargina, permitindo o mesmo controle metabólico com redução significativa na incidência de hipoglicemias graves e noturnas quando comparadas ao período de tratamento com insulina NPH. 
O uso de análogos de insulina de ação prolongada permite um tratamento mais previsível com menos hipoglicemias em comparação à insulina NPH. Esse benefício se deve às suas características farmacocinéticas que possibilitam uma ação prolongada sem pico de ação. A insulina glargina foi o primeiro análogo de longa duração utilizado no tratamento do diabetes. Até o momento, vários ensaios clínicos e estudos observacionais foram publicados em pacientes com DMTl e DM2, avaliando-se o uso da insulina glargina. Porém, estudos em crianças pré-púberes com DMTl são raros, especialmente em crianças menores de seis e oito anos. Alguns estudos selecionaram crianças pré-púberes, que foram arroladas com crianças maiores, para o tratamento com insulina glargina. Porém, apenas um estudo avaliou o uso desse análogo em crianças pequenas (menores de seis anos) (15). As informações provenientes desses vários estudos evidenciam muitos benefícios trazidos pelo uso da glargina em relação às insulinas tradicionais: redução na frequência de hipoglicemias severas, redução na frequência de hipoglicemias noturnas, melhora na qualidade de vida e controle metabólico igual ou superior ao tratamento tradicional (4).

$\mathrm{O}$ uso da insulina glargina reduz a incidência de hipoglicemias, especialmente as noturnas, em crianças maiores e adolescentes. Por outro lado, a hipoglicemia noturna representa uma grande preocupação para os familiares e médicos de crianças pequenas com DMTl. Os nossos resultados confirmam essa vantagem da insulina glargina também em crianças menores de oito anos. O referido benefício também foi observado no estudo de Dixon e cols. (15), em que foram estudadas retrospectivamente crianças diabéticas do tipo $1 \mathrm{com}$ menos de seis anos de idade. No estudo, a ocorrência de hipoglicemia noturna grave foi bem menor durante o tratamento com glargina em comparação ao tratamento com NPH, e a Alc foi semelhante nos dois grupos. Jeha e cols. (16) avaliaram o controle glicêmico em dez crianças com DMTl na fase pré-escolar por meio do CGMS durante três dias. As crianças recebiam duas doses de insulina NPH e lispro. A conclusão foi de que esse esquema terapêutico não permite um controle glicêmico adequado e se associa a hipoglicemias frequentes e prolongadas, especialmente à noite.

Um estudo em crianças com DMTl recentemente diagnosticadas evidenciou uma melhora no controle metabólico dos pacientes tratados com glargina em relação ao grupo tratado com NPH. Em um período de três meses, a Alc ficou estável no primeiro grupo $(6,8 \%$ no início e $6,7 \%$ ao final do tratamento) e aumentou significativamente no grupo com NPH $(6,9 \%$ no início e 7,6\% ao final do tratamento) (9). Outro estudo recente (17) avaliou retrospectivamente a transição do tratamento com insulina NPH para glargina em 62 crianças e adolescentes com média de idade de 12,7 anos. Nesse estudo, a Alc permaneceu estável ao longo de 12 meses, com menor dose diária de insulina, mas sem diferenças no número de hipoglicemias graves. Além disso, relatouse uma maior satisfação com o uso da insulina glargina.

Deja e cols. (7) avaliaram, ao longo de um ano, 70 crianças com DMTl com média de idade de 12 anos. $\mathrm{O}$ tratamento dessas crianças foi modificado de NPH para glargina e os dados foram analisados separadamente em crianças pré-púberes e adolescentes. Observou-se melhora significativa no controle metabólico nos dois grupos, bem como uma tendência na redução dos episódios de hipoglicemias. Porém, nas crianças maiores, a melhora no controle metabólico foi mais significativa. Esses dados confirmam a maior dificuldade no manejo do diabetes em crianças menores. Deiss e cols. (8) constataram uma redução significativa na ocorrência de hipoglicemia assintomática, por meio do CGMS, sem aumentar a Alc. Foram avaliadas 30 crianças com média de idade de 14,2 anos (4,5 a 18,3 anos) antes e oito semanas após a troca do tratamento de NPH pela glargina. No nosso meio, Maia e cols. (18) publicaram seus resultados da mudança de NPH para glargina em pacientes com DMTl e 2, constatando uma redução na Alc de 10,2 para $9,1 \%(\mathrm{p}=0,019)$ associada à redução dos eventos hipoglicêmicos.

$\mathrm{O}$ esquema de transição entre insulina NPH e glargina ainda não está bem estabelecido. Tan e cols. (19) iniciaram a glargina com dose $20 \%$ menor do que a $\mathrm{NPH}$, mas detectaram aumento na ocorrência de hipoglicemias graves durante a primeira semana de tratamento com glargina. $\mathrm{Na}$ experiência aqui relatada, isso não ocorreu. Adotou-se o mesmo critério ao mudar de insulina NPH para glargina e houve redução significativa na ocorrência de hipoglicemias graves desde o início do tratamento. Dixon e cols. (15) reduziram a dose em $50 \%$ no início do uso da glargina e, obviamente, não constataram hipoglicemias graves nesse período de transição.

Como se pode observar, na literatura existe um número significativo de estudos clínicos sugerindo vantagens da insulina glargina em relação à hipoglicemia, especialmente noturna. Mais recentemente, alguns estudos vêm apontando uma melhora metabólica, ilus- 
trada pela melhora da Alc durante o tratamento com a glargina. Porém, observou-se que ainda são escassos os estudos em crianças pequenas (menores que seis e oito anos). Em quase todos os estudos, com exceção de um (15), as informações sobre essa faixa etária estão misturadas com as de crianças maiores, raramente analisadas separadamente. Dessa forma, o presente estudo proporcionou informações consistentes em relação ao tratamento de crianças menores de oito anos, dados pouco encontrados na literatura.

Os nossos dados confirmam a eficácia e a segurança da insulina glargina em crianças menores de oito anos portadoras de DMTl. Foi possível demonstrar uma redução significativa nas hipoglicemias noturnas e hipoglicemias graves, sem modificação na Alc. Portanto, o uso de insulina glargina em crianças com menos de oito anos de idade pode ser considerado uma boa opção terapêutica, especialmente quando o tratamento com insulina NPH estiver associado a hipoglicemias graves e/ou noturnas.

Agradecimentos: à Sanofi Aventis pelo apoio financeiro oferecido para a realização deste estudo.

Declaração: Dr. Balduino Tschiedel é membro do corpo consultivo da Sanofi-Aventis, para a insulina glargina.

\section{REFERÊNCIAS}

1. Gerich J, Bolli G, Becker RHA, Zhu R. Fluctuations of serum insulin levels after single and multiple dosing of insulin glargine. Diabetologia. 2003;46:A271.

2. Hamann A, Matthaei S, Rosak C, Silvestre L, The HOE 901/4007 Study Group. A randomized clinical trial comparing breakfast, dinner or bedtime administration of insulin glargine in patients with type 1 diabetes. Diabetes Care. 2003;26(6):1738-44.

3. Lepore M, Pampanelli S, Fanelli C, Porcellati F, Bartocci L, Di Vincenzo A, et al. Pharmacokinetics and pharmacodynamics of subcutaneous injection of long-acting human insulin analog glargine, NPH insulin, and ultralente human insulin and continous subcutaneous infusion of lispro. Diabetes. 2000;49(12):2142-8.

4. Miles $\mathrm{HL}$, Acerini CL. Insulin analog preparations and their use in children and adolescente with type 1 diabetes mellitus. Paediatr Drugs. 2008;10(3):163-76.

5. Mohn A, Strang S, Wernicke-Panten K, Lang AM, Edge J, Dunger DB. Nocturnal glucose control and free insulin levels in children with type 1 diabetes by use of the long-acting insulin HOE 901 as part of a three-injection regimen. Diabetes Care. 2000;23(4):557-9.
6. Chase HP, Dixon F, Pearson J, Fiallo-Scharer R, Walravens P, Klingensmith $G$, et al. Reduced hypoglycemic episodes and improved glycemic control in children with type 1 diabetes using insulin glargine and neutral protamine Hagedorn insulin. J Pediatr. 2003;143(6):737-40.

7. Deja G, Jarosz-Chobot P, Polanska J. The rate of improvement in metabolic control in children with diabetes mellitus type 1 on insulin glargine depends on age. Exp Clin Endocrinol Diabetes. 2007;115(10):662-8.

8. Deiss K, Kordonouri O, Hartmann R, Hopfenmüller W, Lüke K, Danne T. Treatment with insulin glargine reduces asymptomatic hypoglycemia detected by continuous subcutaneous glucose monitoring in children and adolescents with type 1 diabetes. Pediatr Diabetes. 2007;8(3):157-62.

9. Hassan K, Rodriguez LM, Johnson SE, Taldock S, Heptulla RA. A randomized, controlled trial comparing twice-a-day insulin glargine mixed with rapid-acting insulin alalogs versus standard neutral protamine Hagedotn (NPH) therapy in newly dignosed type 1 diabetes. Pediatrics. 2008;121(3):e466-72.

10. Hathout EH, Fujishige L, Geach J, Ischandar M, Maruo Sem, Mace JW. Effect of therapy with insulin glargine on glycemic control in toddlers, children and adolescents with diabetes. Diabetes TechnolTher. 2003;5(5):801-6.

11. Jackson A, Ternand C, Brunzell C, KleinschmidtT, Dew D, Milla C, et al. Insulin glargine improves hemoglobin $A 1 c$ in children and adolescents with poorly controlled type 1 diabetes. Pediatric Diabetes. 2003;4(2):64-9.

12. Porcellati F, Rosseti P, Pampanelli S, Fanelli CG, Torlone E, Scionti $L$, et al. Better long-term glycaemic control with the basal insulin glargine as compared with NPH in patients with type 1 diabetes mellitus given meal-time lispro insulin. Diabetic Medicine. 2004;21(11):1213-20.

13. Rossetti P, Pampanelli S, Fanelli C, Porcellati F, Costa E, Torlone E, et al. Intensive replacement of basal insulin in patients with type 1 diabetes mellitus given rapid-acting insulin analogue at meal time: a three-month comparison between administration of NPH four times daily and glargine insulin at dinner or bedtime. Diabetes Care. 2003;26(5):1490-6.

14. Schober E, Schoenle E, Van Dyk J, Wernicke-Panten K. Glargine at PSGol. Comparative trial between insulin glargine and NPH insulin in children and adolescents with type 1 diabetes mellitus. J Pediatr Endocrinol Metab. 2002;15(4):369-76.

15. Dixon B, Peter Chase $H$, Burdick J, Fiallo-Scharer $R$, Walravens $P$, Klingensmith $\mathrm{G}$, et al. Use of insuline glargine in children under age 6 with type 1 diabetes. Pediatr Diabetes. 2005;6:150-4.

16. Jeha GS, Karavitti LP, Anderson B, Smith EO, Donaldson S, McGirk TS, et al. Continuous glucose monitoring and the reality of metabolic control in preschool children with type 1 diabetes. Diabetes Care. 2004;27(12):2881-6.

17. Päivärinta $M$, Tapanainen $P$, Veijola $R$. Basal insulin switch from $\mathrm{NPH}$ to glargine in children and adolescents with type 1 diabetes. Pediatr Diabetes. 2008;9(3 Pt 2):83-90.

18. Maia FF, Melo FJ, Araújo LR. Switching of NPH insulin to glargine therapy in a cohort of diabetic patients: observational study. Arq Bras Endocrinol Metabol. 2007;51(3):426-30.

19. Tan CY, Wilson DM, Buckingham B. Initiation of insulin glargine in children and adolescents with type 1 diabetes. Pediatr Diabetes. 2004;5(2):80-6. 